\title{
Modeling the dynamic behavior of railway track taking into account the occurrence of defects in the system wheel-rail
}

\author{
Alexey Loktev ${ }^{1, *}$, Vyacheslav Sychev ${ }^{2}$, Boris Gluzberg ${ }^{3}$ and Ekaterina Gridasova ${ }^{4}$ \\ ${ }^{1}$ Moscow state university of civil engineering, Yaroslavskoye shosse, 26, Moscow, Russia, 129337 \\ ${ }^{2}$ MIIT, Department of Transport Construction, 125190 Moscow, Chasovaya st., 22/2, Russia \\ ${ }^{3}$ VNIIZHT, The integrated office "Path and track facilities", 129626 Moscow, 3-ya Mytischenskaya, 10, Russia \\ ${ }^{4}$ FEFU, Department of Welding Production, 690950 Vladivostok, st. Suhanova, 8, Russia
}

\begin{abstract}
This paper investigates the influence of wheel defects on the development of rail defects up to a state where rail prompt replacement becomes necessary taking into account different models of the dynamic contact between a wheel and a rail. In particular, the quasistatic Hertz model, the linear elastic model and the elastoplastic Aleksandrov-Kadomtsev model. Based on the model of the wheel-rail contact the maximum stresses are determined which take place in the rail in the presence of wheel defects (e.g. flat spot, weld-on deposit, etc.). In this paper, the solution of the inverse problem is presented, i.e., investigation of the influence of the strength of a wheel impact upon rails on wheel defects as well as evaluation of the stresses emerging in rails. During the motion of a railway vehicle, the wheel pair position in relation to rails changes significantly, which causes various combinations of wheel-rail contact areas. Even provided the constant axial load, the normal stresses will substantially change due to the differences in the radii of curvature of contact surfaces of these areas, as well as movement velocities of railway vehicles.
\end{abstract}

\section{Introduction}

Analysis of rail defects in railroad tracks reveals that the most substantial rail defects among detected are operational defects which occur due to the influence of a moving railway vehicle on rails and appear as hairline cracks, delamination and flaking of the rail head material (code 10.1-2, 11.1-2 according to [1]). It is known [1] that during railway operation defects of the codes 10.1-2 and 11.1-2 lead directly to defective rails of the codes 30G.1-2, 30B.1-2 and 21.1-2, which have to be eliminated immediately after their detection $[1,2]$. Horizontal delaminations of the rail head appear due to the presence of nonmetallic impurities (defect of the code 30G.1-2) and transverse cracks, which are visible as light and dark spots in the rail head. Figure 1 shows the fractures formed due to the insufficient contact fatigue strength $\mathrm{f}$ the metal material (defect of the code 21.1-2) and welding joints (defect of the code 26.3).

* Corresponding author: aaloktev@yandex.ru 
Substantial contact stresses between a wheel and a rail arise under large axial loading and high-speed movement of a railway vehicle [3,4]. The contact area under extreme, alternating forces, which cause permanent plastic deformations of the microstructure, is only several millimeters large and rail hardening takes place. Therefore the main safety precaution is to avoid the transition of rails with defects of the codes 10.1-2 and 11.1-2 into fatal defects by carrying out so called rail improvement, which includes weld deposition and rail grinding provided that the defect sizes do not exceed the critical parameters and velocities of railway vehicles are not limited.

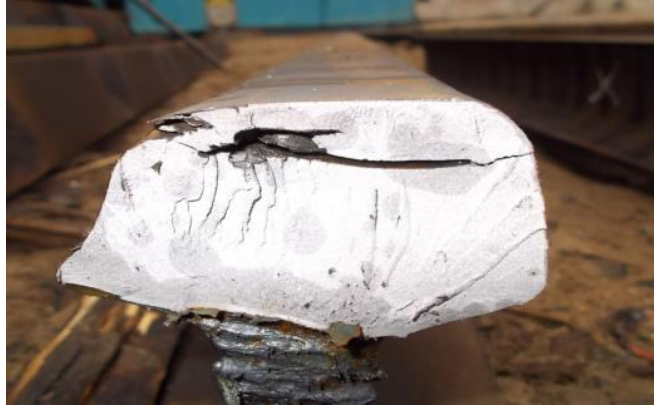

a)

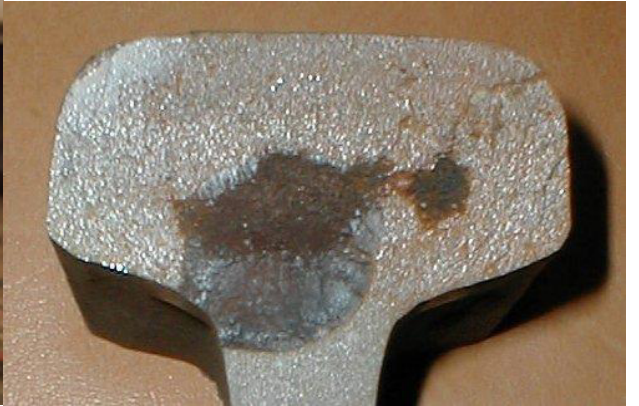

b)

Fig. 1. Rail defects: a) Defect of the code 30G.1 - Transverse cracks in the rail head; b) Defect of the code 26.3 - Transverse cracks in the rail head caused by violation of the joint welding procedure and processing of the welding joints, which led to the rail breakdown after passing of the warranted tonnage in the joining point.

\section{Statement of the problem}

According to the investigations of the Joint Stock Company Railway Research Institute (JSC "VNIIZhT") [3, 4], internal transverse or longitudinal cracks appear in the rail head of new railways at a depth of 3 to $14 \mathrm{~mm}$ from the tread surface (at axial load of rail cars of 150$250 \mathrm{kN}$ ) in the area of nonmetallic impurities, where a high concentration of sign-variable contact stresses is observed. The stress concentrators are located along rails and form microcracks under the influence of wheels of a moving railway vehicle and not necessarily result in formation of internal longitudinal cracks that demand the rail replacement. The following are the reasons for the similar formation and development of the microcracks: incorrect canting of rails and/or wheel defects caused by tread surface irregularities. This leads to the vertical wheel oscillations, as a result of which inertia forces appear in the wheel mass, which in their turn cause additional load on the rails. If this occurs, the impact of a moving wheel with isolated irregularities of the tread surface (i.e. flat spot) takes place, which has a very strong additional influence on the rail.

Figure 2 represents the scheme of a wheel with isolated roughness, i.e. wheel flat, where $l_{n}$, $h_{n}$ - the length and depth of a flat spot; $r$ - the radius of the rolling circle of the wheel; $z_{k}-$ the axis of ordinates of wheel pair trajectory; $l_{n 3}$ - the length of the flat spot; $X$ - the coordinate along the railway path axis; $X A B C$ - the trajectory of a wheel pair.

In the case the flat spot at a wheel surface is found, its depth has to be determined. The shape of the contact area and contact stresses, which can be represented as a distributed load applied along the contact surface, depend on the shape of contact bodies. Neglecting the conical nature of wheel-rail contact we assume for the analysis that the contact between a wheel and a rail can be represented as the contact between two cylinders - one is located perpendicular to the other one. 


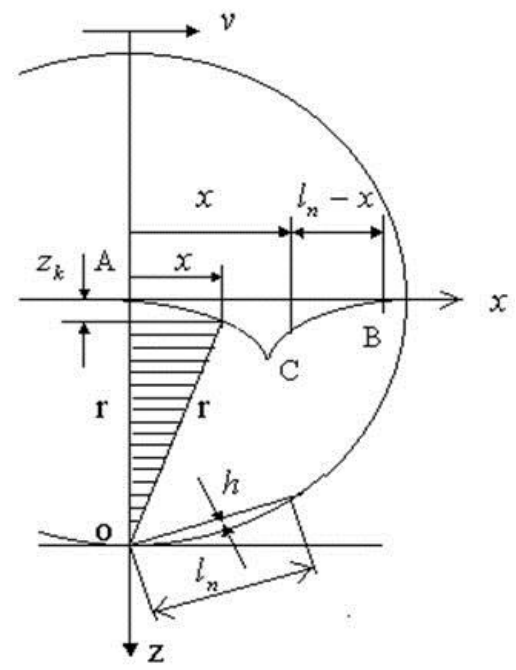

Figure 2. Schematic representation of a wheel with an isolated irregularity - flat spot.

\section{Contact problem}

In the general case the area of the wheel-rail contact has an ellipsoidal shape. Thus, the normal stresses at the wheel-rail boundary depend on the wheel load on the rail, radii of the tread surfaces between wheel and rail and properties of interacting materials.

In this case different contact models can be applied for the analysis, i.e., the quasistatic Hertz model, the linear elastic model, two elastoplastic contact models: the AleksandrovKadomtsev model, the viscoelastic model with an exponential kernel of relaxation, the aggregated model based on the Hertz model and the unloading curve [5-10].

1) The quasistatic Hertz model $[5,7,9]-P=k a^{3 / 2}$,

2) The linear elastic model $[7,8]-P=E_{1}(a-w)$,

3) The viscoelastic model $[6,10]-P(t)=E_{1}(\alpha-w)-\frac{E_{1}}{\tau_{1}} \int_{0}^{t}(\dot{\alpha}-\dot{w}) e^{-\frac{t-t^{\prime}}{\tau_{1}}} d t^{\prime}$,

4) The elastoplastic Aleksandrov-Kadomtsev model [7, 10]

$$
\alpha=\left\{\begin{array}{cll}
b P^{2 / 3}, & d P / d t>0, & P_{\max }<P_{1}, \\
(1+\beta) c_{1}+(1-\beta) P d, & d P / d t>0, & P_{\max }>P_{1}, \\
b_{f} P^{2 / 3}+\alpha_{p}\left(P_{\max }\right), & d P / d t<0, & P_{\max }>P_{1},
\end{array}\right.
$$

5) The model where the force of interaction is divided into loading and unloading steps $[11,12]$. For a loading step - (1), for an unloading step -

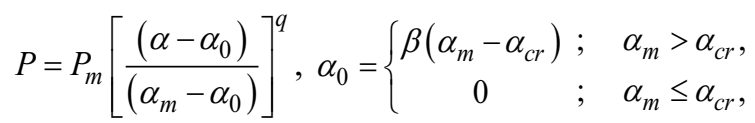

In the expressions (1) - (5), $\alpha$ is the local deformation of wheel and rail materials, $k$ - the coefficient depending on the geometry of contact bodies and elastic properties of their materials, $E_{1}$ - the elasticity modulus of the area of wheel-rail interaction, $w$ - the displacement of the 
lower edge of the rail, $\lambda=5.7, \quad b=\left(\left(9 \pi^{2}\left(k_{1}+k\right)^{2}\right) / 16 R\right)^{1 / 3}, \quad k_{1}=\left(1-\sigma_{1}^{2}\right) / E_{1}$, $k=\left(1-\sigma^{2}\right) / E, \quad \chi=\pi k_{p l} \lambda, \quad P_{1}=\chi^{3}\left(3 R\left(k_{1}+k\right) / 4\right)^{2}, \quad d=1 / 2 \chi R$, $b_{f}=R_{f}^{-1 / 3}\left(3\left(k_{1}+k\right) / 4\right)^{2 / 3}, \quad R_{p}^{-1}=R^{-1}-R_{f}^{-1}, \quad R_{f}=\left(4 / 3\left(k_{1}+k\right)\right) P_{\max }^{1 / 2} \chi^{-3 / 2}$, $\alpha_{p}\left(P_{\max }\right)=(1-\beta) P_{\text {max }}\left(2 \chi R_{p}\right)^{-1}, \beta=0.33, c_{1}=3 \chi^{1 / 2}\left(k_{1}+k\right) / 8, k_{p l}$ - the lowest plastic constant of interacting bodies, $\sigma_{1}, E_{1}$ - the Poisson's ratio and modulus of elasticity of the wheel pair $[2,8], \tau_{1}=\eta_{1} / E_{1}, \tau_{1}$ - the relaxation time in the case of the viscoelastic model, $\eta_{1}-$ the viscous resistance coefficient, $\gamma(0<\gamma \leq 1)$ - the parameter of fractionality, $P_{m}$ - the maximum value of the contact force before the unloading step, $\alpha_{\mathrm{m}}$ - the maximum local deformation of the material in the contact area, $\alpha_{0}$ - the current local deformation value, $q$ and $\beta$ - the experimentally obtained constants, $\alpha_{\mathrm{cr}}-$ the critical local deformation value [13].

For modelling of the dynamic interaction between a wheel and a rail under velocities of up to $90 \mathrm{~km} / \mathrm{h}$ according to [14] one could apply expressions from the contact Hertz theory (1) in engineering analysis. The Hertz theory is valid at the following assumptions: the contact surfaces are homogeneous and isotropic; the frictional forces in the contact area can be neglected; the size of the contact area is negligible compared to the sizes of contact bodies and the characteristic radii of curvature of undeformed surfaces; for the contact problem the solution of a linear elastic half space is used; and the contact surfaces are smooth enough [15].

During the motion of a railway vehicle, the wheel pair position in relation to rails changes significantly, which causes various combinations of wheel-rail contact areas. Even provided the constant axial load, the normal stresses will substantially change due to the differences in the radii of curvature of contact surfaces of these areas, as well as movement velocities of railway vehicles.

\section{The determination of the stresses in the interaction of wheel and rail}

By taking into account defectiveness of wheel pairs and their tread surfaces it cannot be claimed that in the contact area there is only one radius of curvature. Therefore, the Hertz theory would not provide correct results. That is why in this case depending on the defect type, velocity of a railway vehicle and geometry of the upper railway construction (the tangent or the curve of a certain radius) expressions (2) - (5) are more reliable. For the determination of normal contact stresses for a non-conformal contact one could apply the approximation method of nonlinear geometry by using ellipses or carry out the modelling of contact bodies based on the elastic Winkler foundation, where the deformation of surfaces is proportional to the normal contact stresses. The maximum contact stress obtained in this case is 1.3 times higher than that obtained from the Hertz theory $[5,9,11]$. The size of the contact area at the normal wheel-rail geometry as well as the type of contact stresses depend on the normal load between a wheel and a rail, the profiles of a wheel and a rail, the transverse and angular position of the wheel pair on rails as well as the angle of the tread surfaces of rails [3, $7,15]$. According to the shape of the contact area and the type of deformation the intensity of pressure within contact surface is distributed by the semi-elliptic law.

If both cylinders are made from homogeneous material (e.g. carbon steel), the equation for the maximum stress in the contact area between a wheel and a rail $\left(Q_{\max }\right)$ is written as:

$$
Q \max =(3 / 2) \cdot(P / a \cdot b \cdot \pi)
$$

where $P$ - the contact load in $\mathrm{kg}, a$ and $b$ - the semi-axes of the contact ellipse in $\mathrm{cm}$, $(2 / 3) \cdot \pi \cdot a \cdot b \cdot Q_{\max }-$ the volume of the semi-ellipsoid of stresses, which equals to the force $P$ 
in $\mathrm{kg}$ according to the law of equality between a force and its reaction force). The values of the semi-axes of ellipse of the contact area in the case of two cylinders are given by:

$$
A=\left(1 / 2 \cdot R_{2}\right) \text { and } B=\left(1 / 2 \cdot R_{1}\right) \text {, }
$$

where $R_{1}$ and $R_{2}$ - the radii of curvature of the wheel-rail surfaces $\left(R_{2}>R_{1}\right)$. Hence, $A / B=2 \cdot R_{1} / 2 \cdot R_{2}=R_{1} / R_{2}$.

Thus, the smaller radius is denoted as $R_{1}$ - the curvature of the rail head surface, and the bigger as $R_{2}$ - the wheel radius. In this case the maximum contact stress between a wheel and a rail is as follows:

$$
Q_{\max }=\alpha \cdot{ }^{3} \sqrt{ } P \cdot E^{2} / R^{2},
$$

where $\alpha$ - the coefficient depending on the ratio $R_{1} / R_{2}$, taken for the calculation as $0.586 ; P$ - the maximum dynamic vertical force of a wheel pressure on rail in $\mathrm{kg} ; E$ - the modulus of elasticity of rail's steel, which equals to $2.1 \cdot 10^{6} \mathrm{~kg} / \mathrm{cm}^{2}$. In the case of rail wear in the middle of the rail head, the radius becomes $320 \mathrm{~mm}$, and at the distance of $20 \mathrm{~mm}$ from the longitudinal rail axis towards both sides $-100-200 \mathrm{~mm}$, which is also corroborated by earlier investigations $[3,4,6,7,14]$.

If both a wheel and a railway track possess defects - flat spot and rail head wear, respectively - the contact spot shifts to the edge of the rail head. In these cases $[3,5,7,9$, $11,12,13]$ the maximum impact force of a wheel with a flat spot on rail is determined as:

$$
P=V_{u d} \cdot V_{m_{p r}} \cdot G_{k t}
$$

where $V_{u d}$ - the velocity of the striking point of the wheel, $\mathrm{cm} / \mathrm{s}$ (Fig. 1); $m_{p r}$ - the reduced mass of the rail, $\mathrm{kg} \cdot \mathrm{s}^{2} / \mathrm{cm} ; G_{k t}-$ the rigidity of the wheel-rail contact $0.5 \cdot 10^{6} \mathrm{~kg} / \mathrm{cm}$. The reduced mass of the rail is calculated according to the following formula [8]:

$$
m_{p r}=(1,5 \div 2,3) \cdot m_{r} / k
$$

where $m_{r}$ - the mass per unit length of the rail $0.65 \mathrm{~kg} / \mathrm{cm} ; k$ - the relative rigidity factor of the rail support on broken stone ballast and at rail type R-65 is assumed to be $0.01 \mathrm{~cm}^{-}$ ${ }^{1} .981$, since the sleeper density is $1840-2000$ pieces per $1 \mathrm{~km}$ and the rail wear is from 3 to $6 \mathrm{~mm}$ :

$$
V_{u d}=V \cdot L_{0} / R_{k}
$$

where $V$ - the movement velocity, $\mathrm{cm} / \mathrm{s} ; L_{0}$ - the length of the wheel flat spot, $\mathrm{cm} ; R_{k}-$ the radius of the wheel, $\mathrm{cm}$.

\section{Conclusions}

The sorting out of defective wheels with flat spots of $1.5-2 \mathrm{~mm}$ in a locomotive depot makes $80 \%$ of all wheel defects. Therefore, one of the most important tasks in assuring the reliable functioning of the railway track is the diagnostics of causes and the determination of possibilities for reduction of dynamic forces from wheels of a moving railway car on the rails. Based on the impact force of a defect wheel upon rail (e.g. (3)) the value of the wheel defect can be determined, i.e. the inverse problem to the described above can be solved. This inverse problem - the determination of the value of a wheel defect (e.g. the size of a flat spot) from the impact force upon rail - can be solved by a system of automated diagnostics of a railway vehicle's technical condition. The system operation is based on measuring of vertical forces by determination of rail web deformations and sorting out of wheels by using force criteria of the evaluation of wheels roughness. 


\section{References}

1. "Normative and technical documentation/classification of rail defects/catalogue of rail defects/signs of a defective and fatal cropped rails" (NTD/TSP-1, 2, 3 - 93). Moscow: TRANSPORT. 126 p. (1993)

2. Technical specifications for works on reconstruction (modernization) and repair of railway tracks. The order of JSC "RZD" from 18.01.2013 No. 75r.

3. L. G. Krysanov, A. Yu. Abdurashitov. Path and track facilities, 6, 26-30 (1988)

4. Yu. Abdurashitov, M. N. Georgiev, L. G. Krysanov. Reliability of rails in various climatic conditions. Moscow: VNIIZHT, 138 p. (1987)

5. Yu. N. Mazov, A. A. Loktev, V. P. Sychev. Vestnik MGSU, 5, 61-73 (2015)

6. A.A. Loktev. Acta Mech., 222(1-2), 17-25 (2011)

7. A.A. Loktev. I. J. of Eng. Science, 50, 1, 46-55 (2012)

8. A.A. Loktev, A.V. Sycheva, V.V. Vershinin. Proceeding of the 2014 International Conference on Theoretical Mechanics and Applied Mechanics, Venice, Italy, March 1517, 16-19 (2014)

9. A.Y. Kogan, A. A. Vergoten. Calculation of the impact on road wheelsets with the slider. Moscow: TRANSPORT, 224 p. (1984)

10. A.A. Loktev, G.N. Talashkin, K.D. Stepanov. Transport of the Rus. Fed. 2-3(63-64), 4044 (2016)

11. M. Agostinacchio, D. Ciampa, M. Diomedi, S. Olita. J. of Modern Transportation, 21. 3. 169-181. (2013)

12. R. Olsson, M.V.Donadon, B.G. Falzon. I. J. of Solids and Struct., 43, 3124-3141 (2006)

13. S. Abrate. Compos. Struct., 51, 129-138 (2001)

14. P. Chen, J. Xiong, Z. Shen. Mech. of Materials, 40, 183-194 (2008)

15. A.P. Christoforou, A.A. Elsharkawy, L.H. Guedouar. Comp. and Struct., 79, 2607-2619 (2001) 